\title{
Positioning in Dynamic Testing of Slender Bridges
}

\author{
Alexander Tesar ${ }^{1}$, Jan Bencat ${ }^{2}$ \\ ${ }^{1}$ Institute of Construction and Architecture, Slovak Academy of Sciences, Bratislava, Slovak Republic; ${ }^{2}$ Department of Structural \\ Mechanics, University of Zilina, Komenskeho, Zilina, Slovak Republic. \\ Email: usarate@savba.sk, bencat@fstav.utc.sk
}

Received June $5^{\text {th }}$, 2011; revised July $6^{\text {th }}, 2011$; accepted July $18^{\text {th }}, 2011$.

\begin{abstract}
The positioning combined with multi-functioning and interactive mechanics in dynamic testing of slender bridges are treated in present paper. The approach takes into account multiple functions in dynamic testing of slender bridges constructed of thin-walled structural members with their hierarchical configuration. Theoretical, numerical and experimental in situ assessments of the problem are presented. Some results of the application in situ are submitted.
\end{abstract}

Keywords: Bridges, Deformation Positioning, Dynamic Testing, Interactive Mechanics, Fourier Transformation, Multi Functioning, Optimization, Virtual Monitoring, Wave Propagation

\section{Introduction}

One of interesting features of natural organisms from a design point of view is the co-existence of multiple functions in a single component. Natural organisms are the set-ups with many sub-systems being integrated together. The nature contains countless elegant and highly multifunctioning systems that are of great relevance to human design [1].

Such integration and multi-functioning appear also in dynamic testing and virtual monitoring of deformation positioning of slender bridges used in present structural engineering. Some previous studies of the problem are summed up, for example, in references [2,3]. During development of slender bridges the engineers have adopted a design philosophy of integrating various design functions together. Some of the issues considered in their design are mentioned as follows:

\subsection{Multi-Function 1-Optimal Loading Control}

The loads can be varied by suitable geometry and shape of structural members adopted. For example, the wind turbulences produce a strong vortex wake associated with aerodynamic drag force experienced by structural member. Depending on the wind speed and the cross-section's shape, the shedding of vortices is more or less regular with shedding periods inversely proportional to the wind speed. In resonant conditions structure's oscillations control the rhythm of the vortex shedding appearing. Aside the known vortex trail type excitation the more general types of aerodynamic forcing appear there. Aeroelastic forces proportional to the movement of the structure result in self-induced vibrations at high wind speeds. In design is to be avoided that absolute value of negative aerodynamic damping force exceeds the positive mechanical damping force producing oscillatory torsional or across-wind flexural mode instability.

\subsection{Multi-Function 2-Optimal Structural Layout}

The layout of structural members in slender bridge is optimal for generating an efficient flow of forces. Besides an optimal layout the bridges also have optimal material and shape properties being structurally efficient for resisting loads. The single member is in a continuous contact with other members and the modeling takes into account all interactions appearing.

\subsection{Multi-Function 3-Optimal Fail-Safe Mechanisms}

Modern bridges are equipped with embedded self-healing mechanisms that are activated when, for example, a crack in concrete, steel or wood material develops. The self-healing cells in connections of thin-walled members consist of interacting structural members being activated when the material is overstressed. The self-healing cells are small enough so that overall structural properties of the structure are not significantly compromised. The failsafe mechanisms are adopted in hierarchical configuration. If the elements are overloaded they will unzip from 
adjacent members before serious damages occur. Once unzipped the elements can be re-zipped together by simple load and stress redistribution. The large number of separate zipping mechanisms ensures that the structure will unzip very close to the limit point of overload, thus causing minimum damage.

\subsection{Multi-Function 4-Tuned Behaviour Control}

New devices for tuned behavior control are also multifunctionally adopted in slender bridges. For example, to ensure the satisfactory interaction between such materials as fiber composites, wood, steel or concrete, the shear connectors have to be placed in the areas of concentrated load introduction. Such tuning facilities are adopted with regard to three main items as are the changes in stiffness, moment resistance and rotation capacity (ductility). Tuned vibration with control joints also contributes to multifunctioning in slender bridges subjected to vibration forcing.

Some approaches dealing with multi-functioning in dynamic testing and virtual monitoring of slender bridges are considered in this paper. Dynamic testing is applied to slender bridges as part of their inspection and assessment. Such technique is adopted with emphasis on data processing and experimental set-up involved in the evaluation of the dynamic amplification factor and dynamic properties of the bridge. Vertical accelerations, displacements and strains are measured under ambient traffic. Such approaches are observed by experimental and analytical studies in bridge dynamics (see references [2,3]). Most dynamic tests are carried out to evaluate dynamic properties (vibration frequencies and mode shapes, power spectral density, linear and nonlinear time response, etc.) using direct input force mechanisms (shakers, running trucks, impact testing) and modal analysis techniques. These properties are used for finite element model calibration and as a reference in monitoring of dynamic behavior of bridge structures.

\section{Structural Assessment}

Primary point in structural assessment is the evaluation of the dynamic amplification parameter DAF generated by traffic loads on bridges. The parameter is specified by

$$
R_{d y n}=R_{\text {sta }}(1+D A)
$$

where $R_{d y n}$ is the maximum response of the bridge generated by a moving load (maximum dynamic response) and $R_{\text {sta }}$ is the maximum response generated by the same load applied statically (maximum static response). The term $(1+D A)$ is called the dynamic amplification factor (DAF) and is the amount by which the static effects are increased by bridge-vehicle interaction. For design pur- poses dynamic load allowance coefficient DLA is adopted, based on statistical values of DAF obtained by dynamic testing and analysis. This coefficient is usually related to first vibration frequency of the bridge or to its span length.

The analysis is based on the the presumption of propagating waves with efficient exchange of potential and kinetic energy appearing. The bridge is radiated by the waves initiated by traffic or other load impacts and the response appearing is transmitted into all structural parts studied.

The wave propagation has two physical aspects-the source of waves and the medium where the waves are running. The waves initiated in the source are filtered during propagation in the bridge and are specified by spectral evolution. The diffraction of waves appears there in the inhomogeneities of the bridge.

The spectral evolution is based on following definitions:

1) Each stationary function $x(t)$ is interpreted in integral form by

$$
x(t)=\int \mathrm{e}^{i \omega t} \mathrm{~d} A(\omega)
$$

with $A(\omega)$ as orthogonal complex process and with frequency $\omega$.

2) The linear transformation $y(t)$ of the function $x(t)$ is given by

$$
Y(t)=\int H(i \omega) \mathrm{e}^{i \omega t} \mathrm{~d} A(\omega)
$$

with $H(i \omega)$ as admittance function and $\mathrm{e}^{i \omega t}$ as unit impulse adopted.

3) The spectral densities $S_{x}$ and $S_{y}$ of functions $x(t)$ and $y(t)$ are related by

$$
S_{y}(\omega) / S_{x}(\omega)=|H(i \omega)|^{2}
$$

The structure is defined by wave numbers $r_{i}(\omega)$ with longitudinal and shear waves.

The stationary waves are emitted from the source with amplitude $F\left(\omega, z_{o}\right)$, i.e., $z=z_{o}$. The wave superposition is given by

$$
w_{i}(t, z)=\int \mathrm{e}^{-i \omega t} \mathrm{e}^{i r\left(\omega_{z}\right)} \mathrm{d} F\left(\omega, z_{o}\right)
$$

The structural inhomogeneity is touched by propagating waves. The response spectrum for such case is given by

$$
S_{R}(\omega, 0)=S\left(\omega, z_{o}\right)|H(\omega, 0)|^{2} \mathrm{e}^{-2 \operatorname{Im}[r(\omega)]}
$$

Response spectrum obtained is the basis for specification of physical parameters in virtual monitoring of bridges, with adoption of the identification approach described below.

\section{Identification Approach}

Assumed is the system forcing given by white noise 
processes $\mathrm{e}_{x}\left(t_{k}\right)$ and $\mathrm{e}_{m}\left(t_{k}\right)$. Discrete time equivalents $\Delta x\left(t_{k+1}\right)$ and $\Delta y\left(t_{k+1}\right)$ are given by

$$
\begin{gathered}
\Delta x\left(t_{k+1}\right)=F \Delta x\left(t_{k}\right)+B \mathrm{e}_{x}\left(t_{k}\right) \\
\Delta y\left(t_{k+1}\right)=\left(G_{1}+G_{2}\right) A \Delta x\left(t_{k}\right)+\mathrm{e}_{m}\left(t_{k}\right)
\end{gathered}
$$

where the state vector $\Delta x$ contains the members related to the bridge with possible stochastic properties and so do the system matrices $F, B, A, G_{1}$ and $G_{2}$ (see references [4] or [5]).

Covariance matrices for forcing are denoted $R_{x}\left(t_{i}\right)$ and $R_{x m}\left(t_{i}\right)$. The covariance matrices $Y\left(t_{i}\right)$ of the process $y\left(\mathrm{t}_{\mathrm{k}}\right)$ with system matrices of the model are given by

$$
Y_{K}\left(t_{i}\right)=G F_{K-1} M\left(t_{i}\right)
$$

with

$$
M\left(t_{i}\right)=F R_{x}\left(t_{i}\right) G^{T}+B R_{x m}\left(t_{i}\right)
$$

The Hankel matrix established by covariance matrices $Y_{k}\left(t_{i}\right)$ is used in identification and is given by operations:

1) Form the Hankel matrix of the problem studied.

2) Compute the singular value decomposition of the Hankel matrix by

$$
H(p)=U T^{2} V^{T}
$$

where $U$ and $V$ are orthogonal matrices and $T$ is diagonal and semidefinite matrix of singular values.

3) System matrices are given by

$$
\begin{gathered}
F=T^{-1} U^{T} H(p) V \\
M=T V^{T} E_{n} \\
G=E_{n} U T
\end{gathered}
$$

with

$$
E_{n}=\left[I_{n} \cdots 0 \cdots 0 \cdots 0\right]
$$

4) Solve unsymmetric eigenvalue problem established from the $(m \times m)$-upper left submatrix of the $(n \times n)$ system matrix $F$.

5) Compute modal parameters from eigenvalues and modes obtained.

6) Repeat the operations from step 4 for increasing system submatrix.

Before application of identification approach the filtering of data is required. The type of filter applied depends on actual forcing conditions appearing.

\section{Ultimate Response}

The Fourier integral transformation combined with the FETM-aproach [6] is adopted for dynamic testing. Considered is the load $P_{j}(t)$ moving with velocity $c$ on the bridge studied. The load is located in time $\mathrm{t}$ in nodes $j=1$,
$2, \cdots, n$. The response in node $\mathrm{i}$ is given by $X_{i}(t)(i=1$, $2, \cdots, m)$. The symbol $g_{i j}(\omega)$ specifies the spectral response function in node i due to input unit impulse $\mathrm{e}^{i \omega t}$. There holds

$$
X(t)=\int g(t) P(t) \mathrm{d} t
$$

with vectors $X(t)$ and $P(t)$ of functions $X_{i}(t)$ and $P_{i}(t)$, respectively, and with $g(t)$ as $(m \times m)$-matrix of functions $g_{i j}(\omega)$. The response is given by

$$
M_{Y}(\omega)=N(\omega) M_{p}(\omega)
$$

where $N(\omega)$ is the transfer function of the bridge and $M_{Y}(\omega)$ is ultimate transform of forcing function $M_{P}(\omega)$. The function of structural response $N(\omega)$ defines spectral characteristics of the bridge and specifies amplitude and phase shift of the response related to the components of the forcing assumed.

The approach allows the consideration of all structural parameters appearing. In updated Lagrangian formulation of motion geometric nonlinearities are implemented into coordinate transformations of the microelement mesh used. The physical nonlinearities are analysed on the level of macroelement mesh adopted.

\section{Structural Optimization}

Optimization is adopted as a selection of testing parameters in monitoring of bridges (see references [7-11]). The selection of testing parameters is subjected to following constraints:

- Geometric constraints-minimum and maximum areas, dimensions and rigidities of thin-walled members adopted,

- Stress constraints-maximum allowable stress,

- Displacement constraints-minimum and maximum deformations, rotations and displacements,

- Resonance, stability and fatigue ultimate limits.

Such items specify the regional constraints and can be applied for all loads occurring. They are represented by constraint hypersurfaces. Stress, deformations and resonance, stability or fatigue ultimate limits are nonlinear functions of testing variables adopted. The constraint hypersurfaces are nonlinear functions of such variables. If virtual testing point lies above constraint surfaces then stress or displacements in regional constraints are within specified limits.

The optimization is stated as follows: Find the geometric testing variables $Y$ and the cross-sectional design variables $X$ or $Z$ (as other types of variables) given by objective function

$$
W=F(X, Y, Z) \rightarrow \min
$$

geometric constraints 


$$
\begin{aligned}
X^{L} & \leq X \leq X^{U} \\
Y^{L} & \leq Y \leq Y^{U} \\
Z^{L} & \leq Z \leq Z^{U}
\end{aligned}
$$

stress constraints

$$
\sigma^{L} \leq \sigma \leq \sigma^{U}
$$

displacement constraints

$$
r^{L} \leq r \leq r^{U}
$$

resonance, stability and fatigue constraints

$$
\gamma^{L} \leq \gamma \geq \gamma^{U}
$$

where the superscripts $L$ und $U$ denote the lower and upper bounds, respectively. The terms $\sigma, r$ and $\gamma$ are the vectors of stress, displacements and resonance, stability or fatigue limits adopted. Such limits are implicit functions of testing approach given by following steps:

1) Assume an initial structural geometry,

2) Optimize testing variables for given geometry by satisfying Equations (18)-(24),

3) Modify the variables,

4) Repeat the steps 2 and 3 until the results are obtained.

The number of testing variables is to be reduced by specifying all above parameters in terms of a small number of independent variables. In order to reduce the number of testing variables is used a coarse grid in the space of geometric variables, so that only a small number of $X, Y$ or $Z$ values is to be considered. In order to optimize the $X, Y$ and $Z$ variables, one of known unconstrained minimization techniques (see references [6,9-16]) is to be used.

\section{Application}

Studied is dynamic testing in monitoring of the Old Bridge crossing Danube in Bratislava, Slovakia ([4,5,1719]). The span of the bridge field studied is $75.82 \mathrm{~m}$ and the width of the bridge is $7.00 \mathrm{~m}+2.39 \mathrm{~m}$ (pedestrian path) (see Figures 1-4).

In scope of dynamic testing was studied the selection of testing parameters with following constraints:

- Geometric constraints,

- Stress constraints,

- Displacement constraints-maximum midspan deformation of the bridge was limited by $1 / 400$ of the span,

- $\quad$ Resonance, stability and fatigue ultimate limits were assumed in accordance with valid standards.

The forcing was made by smooth runs of truck with weight $169.5 \mathrm{kN}$ and with velocities 5 - $68 \mathrm{~km} / \mathrm{h}$.

Calculations were based on above theoretical approach and were confronted with experimental results in order to develop the model for the analysis. For calculation was adopted the software NASTRAN, with development of the models with optimal mesh in the FEM approach and taking into account actual boundary conditions of the bridge studied.

Calculated were 100 natural frequencies of vibration $f_{(j)}$ with corresponding modal analysis and with determination of corresponding 20 modes of natural vibration $w_{(j)}$. The first 3 modes of vibration are plotted in Figures 5-7.

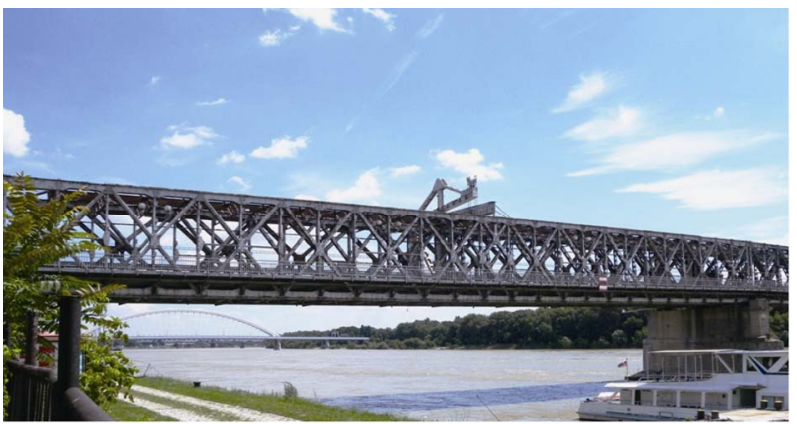

Figure 1. Studied span of the old bridge crossing Danube in Bratislava.

\section{ELEVATION}

\section{OLD BRIDGE CROSSING DANUBE IN BRATISLAVA}

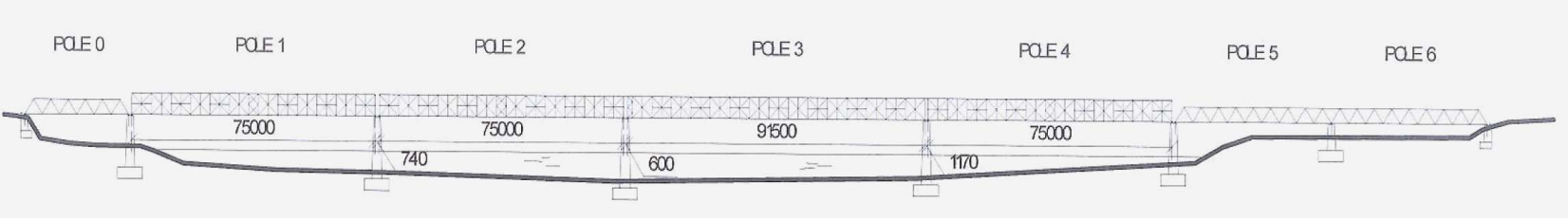

Figure 2. Elevation of the bridge. 


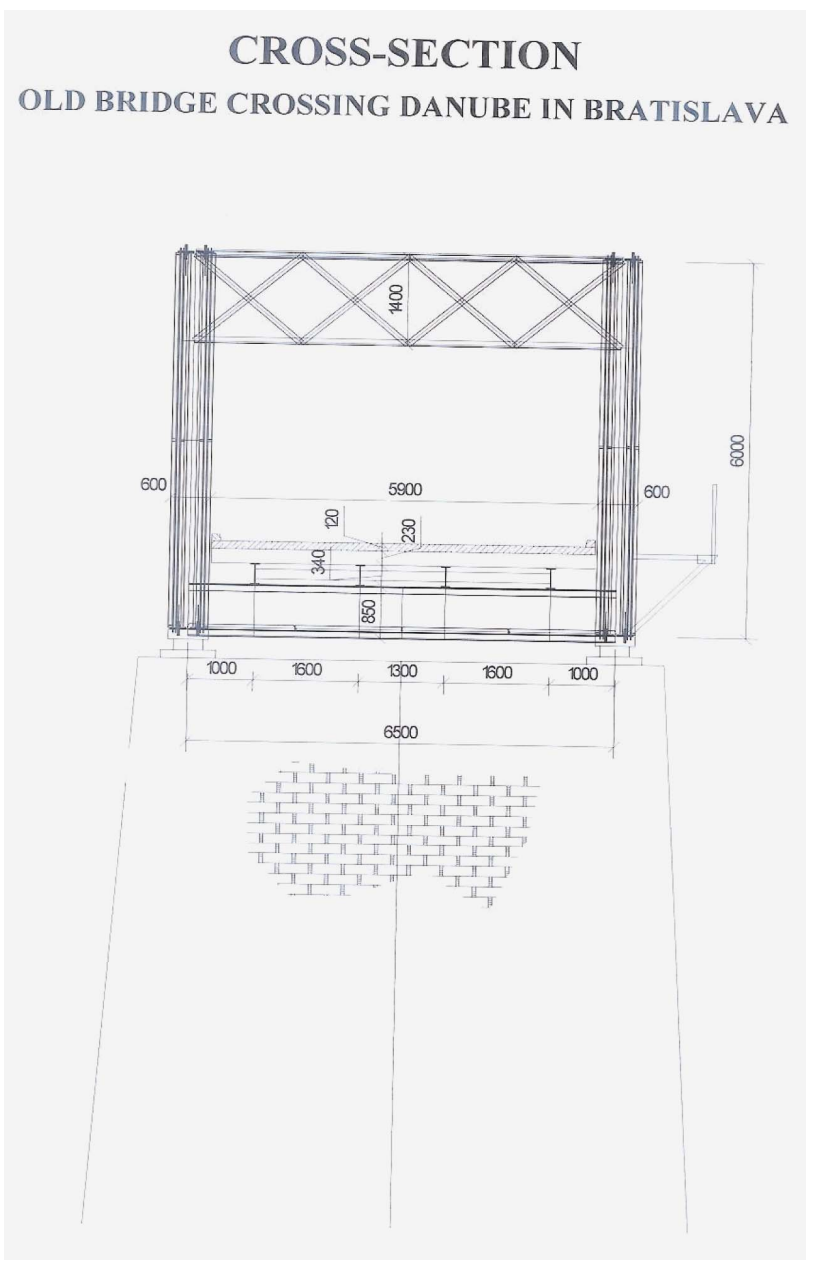

Figure 3. Cross-section of the bridge.

Corresponding natural frequencies are summed up in Table 1. The comparison of calculated and measured dynamic coefficients of the bridge at various truck velocities is submitted in Table 2.

Significant goal was the assessment of actual stiffness of the bridge on the basis of calculated natural frequencies and logarithmic decrements of damping compared with corresponding actual values measured in situ.

The frequency analysis submitted the information required and was made in scope of all measurements done. Obtained were 112 spectra for specification of 9 dominant natural frequencies (3 for flexure in vertical direction, 4 for flexure in horizontal direction, one torsional frequency and one frequency concerning the vibration of pedestrian path). The comparison calculation vs measurement for models without and with consideration of measured corrosion of the bridge is given in Table 3.

In Table $\mathbf{4}$ are summed up the dominant frequencies specified on the basis of the frequency analysis of the bridge. First frequencies in rows correspond to maximal
Table 1. Natural frequencies of the bridge.

Mode Natural frequency $f_{(j)}[\mathrm{Hz}] \quad$ Direction of vibration

\begin{tabular}{ccc}
\hline 1 & 1.630 & Horizontal, flexure \\
2 & 2.349 & Vertical, flexure \\
3 & 3.339 & Horizontal, flexure \\
4 & 3.438 & Horizontal, longitudinal \\
5 & 4.062 & Local vibration \\
6 & 5.399 & Horizontal, flexure \\
7 & 5.447 & Vertical, flexure \\
8 & 6.266 & Torsion \\
9 & 7.693 & Horizontal, flexure \\
10 & 8.724 & Vertical, flexure \\
11 & 9.752 & Vertical, flexure \\
12 & 9.851 & Vertical, flexure \\
13 & 11.048 & Vertical, flexure \\
14 & 11.585 & Vertical, flexure \\
15 & 12.073 & Torsion \\
16 & 12.280 & Horizontal, flexure \\
\hline
\end{tabular}

date in power spectrum $S_{D}(f)$ and the values in brackets represent frequencies with minor significance. In Table 3 is submitted also the comparison of natural frequencies found by numerical and experimental assessment.

Besides spectral analysis was made also the amplitude analysis submitting the basic stochastic parameters for the assessment. Statistical data obtained with 14 measurements on 4 channels submitted 504 values for amplitude analysis of the bridge. On the basis of extreme amplitudes the dynamic coefficients $\delta_{O B S}$ were specified together with corresponding logarithmic decrements of damping. Evaluated were FFT-spectra (Fast Fourier Transformation) adopted for specification of 6 dominant frequencies as reference data in the assessment. Simultaneously was made the spectral analysis for specification of coherence functions of the bridge. Some results are summed up in Figures 8-10 as well as in Tables 2 and 3. The damping coefficients of the bridge vary in scope 0.016 0.038. Amplitude analysis has submitted following maximal midspan deflections: $15.11 \mathrm{~mm}$ (flexure-vertical direction), $6.77 \mathrm{~mm}$ (flexure-horizonal direction) and $16.13 \mathrm{~mm}$ (roadway-vertical direction). 


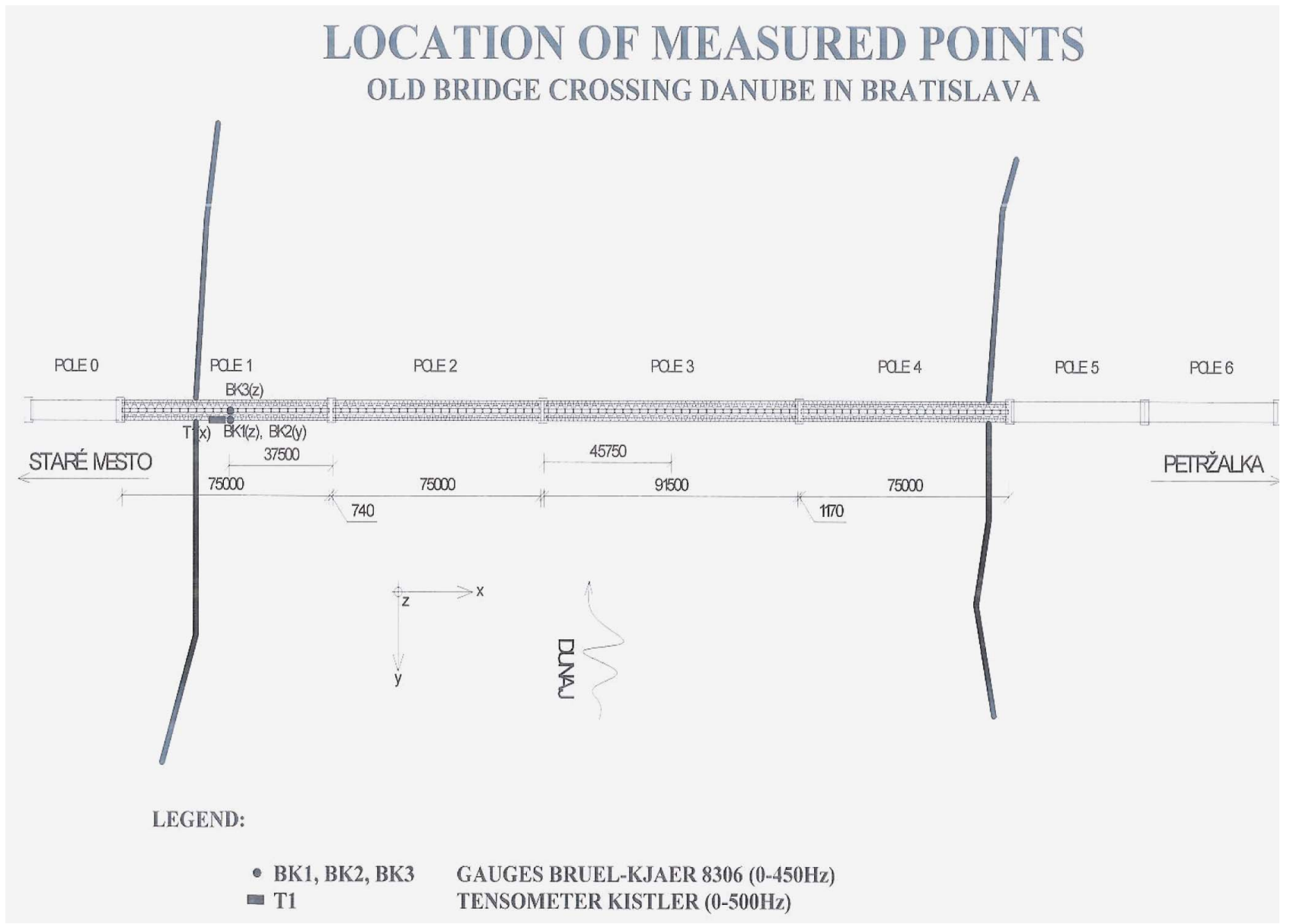

Figure 4. Location of measured points.

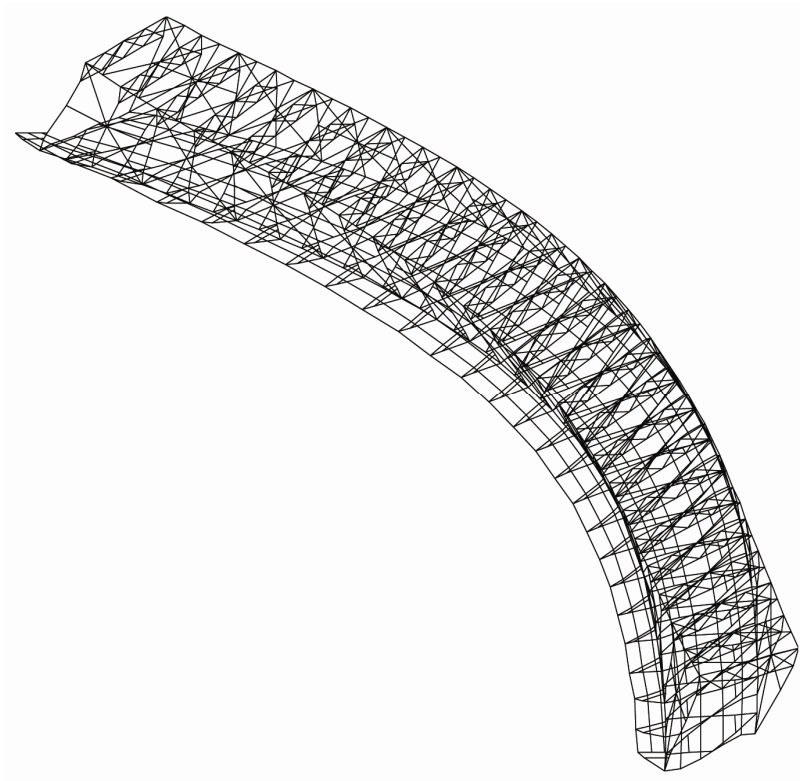

Figure 5. Old bridge in Bratislava-mode Nr. 1, $f_{(1)}=1.630$ Hz.

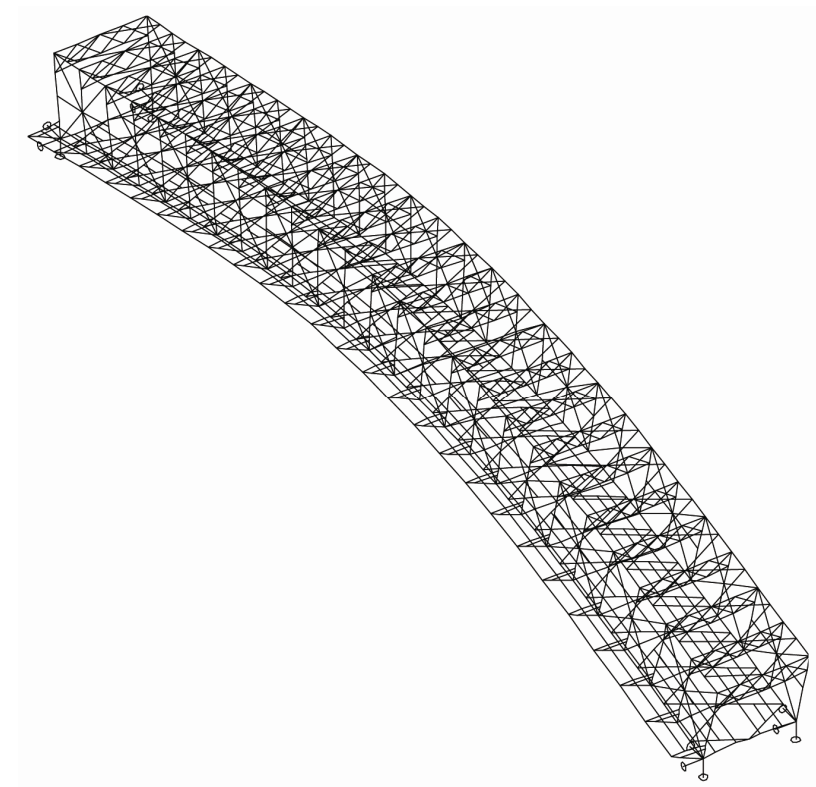

Figure 6. Old bridge in Bratislava-mode Nr. 2, $f_{(2)}=$ $2.349 \mathrm{~Hz}$. 


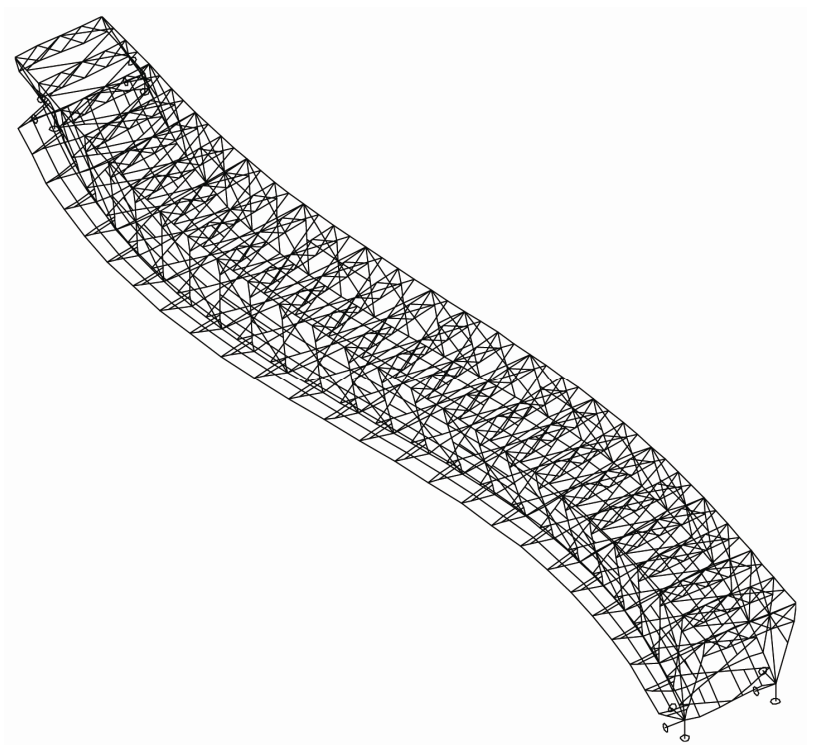

Figure 7. Old bridge in Bratislava-mode Nr. 3, $f_{(3)}=$ $3.339 \mathrm{~Hz}$.

Table 2. Dynamic coefficients of the bridge.

\begin{tabular}{|c|c|c|c|c|}
\hline \multicolumn{3}{|c|}{$\begin{array}{c}\text { Old Bridge crossing Danube in } \\
\text { Bratislava }\end{array}$} & \multicolumn{2}{|c|}{$\begin{array}{c}\text { Dynamic coefficient Smooth } \\
\text { runs }\end{array}$} \\
\hline \multirow{2}{*}{ Run Nr. } & \multirow{2}{*}{ Direction } & \multirow{2}{*}{$\boldsymbol{V}[\mathrm{km} / \mathrm{h}]$} & 1.span & 1.span \\
\hline & & & $\delta_{\text {OBS }}(\mathrm{T} 1)$ & $\delta_{E X P}(\mathrm{~T} 1)$ \\
\hline 1 & BA-PE & 8 & 1.096154 & 1.005865 \\
\hline 2 & PE-BA & 9 & 1.244083 & 1.014889 \\
\hline 3 & BA-PE & 18 & 1.239645 & 1.014618 \\
\hline 4 & PE-BA & 18 & 1.553254 & 1.033749 \\
\hline 5 & BA-PE & 27 & 2.337278 & 1.081574 \\
\hline 6 & PE-BA & 27 & 1.254438 & 1.015521 \\
\hline 7 & BA-PE & 34 & 1.366864 & 1.022379 \\
\hline 8 & PE-BA & 39 & 1.627219 & 1.03826 \\
\hline 9 & BA-PE & 54 & 3.017751 & 1.123083 \\
\hline 10 & PE-BA & 45 & 2.736686 & 1.105938 \\
\hline 11 & BA-PE & 54 & 2.988166 & 1.121278 \\
\hline 12 & PE-BA & 54 & 2.233728 & 1.075257 \\
\hline 13 & BA-PE & 45 & 2.899408 & 1.115864 \\
\hline 14 & PE-BA & 68 & 2.943787 & 1.118571 \\
\hline
\end{tabular}

All details concerning the calculations and measurements made (deflections, sensors, software, technical equipment, repeatability, etc.) are summed up in References $[4,5]$.

\section{Conclusions}

Some approaches for dynamic testing of slender bridges are submitted. The procedures for dynamic testing were
Table 3. Comparison calculation vs measurement.

\begin{tabular}{|c|c|c|c|c|}
\hline \multirow{2}{*}{$\begin{array}{c}\text { Frequency } \\
\text { Nr. }\end{array}$} & \multicolumn{2}{|c|}{ Calculation $^{*}$} & \multirow{2}{*}{$\begin{array}{c}\begin{array}{c}\text { Experimental } \\
\text { analysis }^{* *} \text { ) }\end{array} \\
\text { Span } 1\end{array}$} & \multirow{2}{*}{ Mode } \\
\hline & Model 1 & Model 2 & & \\
\hline 1 & 1.92 & 1.630 & 1.79 & V-O \\
\hline 2 & 2.75 & 2.349 & 2.57 & Z-O \\
\hline 3 & 3.91 & 3.339 & $3 ., 77$ & $\mathrm{~V}-\mathrm{O}$ \\
\hline 4 & 4.21 & 3.438 & - & OS \\
\hline 5 & 4.78 & 4.062 & 4.27 & LOCAL \\
\hline 6 & 6.59 & 5.399 & 5.12 & $\mathrm{~V}-\mathrm{O}$ \\
\hline 7 & 6.65 & 5.447 & 5.51 & Z-O \\
\hline 8 & 7.40 & 6.266 & 6.50 & TOR \\
\hline 9 & 9.05 & 7.693 & 7.68 & $\mathrm{~V}-\mathrm{O}$ \\
\hline 10 & 10.54 & 8.724 & 8.73 & Z-O \\
\hline 11 & 11.56 & 9.752 & - & Z-O \\
\hline 12 & 11.64 & 9.851 & - & Z-O \\
\hline 13 & 13.01 & 11.048 & - & Z-O \\
\hline 14 & 13.59 & 11.585 & - & Z-O \\
\hline 15 & 14.12 & 12.073 & - & TOR \\
\hline 16 & 14.31 & 12.280 & - & V-O \\
\hline
\end{tabular}

"FEM model 1-without considering of corrosion of load bearing members model 2-with consideration of corrosion (decrease of material $1 \mathrm{~mm}$ ) and with dead weight of connecting members; ${ }^{* *}$ dominant frequencies with significant influence in spectral power density (PSD) data with non-significant energy influence in power spectral density.

LEGEND:

$V-O$-horizontal flexural vibration

$Z$ - $O$ - vertical flexural vibration

TOR - torsional vibration

$O S$ - horizontal longitudinal vibration

applied in order to evaluate dynamic properties and corresponding amplification factors. Such tests are part of bridge inspection in order to establish a basis for standard procedures in dynamic testing. Tests carried out under traffic on the bridges are preferred. Some results obtained are presented.

Specified were frequencies of vibration with modal analysis and with comparison of calculated and measured dynamic coefficients of the bridge studied. Significant was the assessment of the stiffness of the bridge on the basis of logarithmic decrements of damping compared with actual values measured in situ. Such analysis submitted the information required and was made in scope of all measurements done. Obtained were frequency 

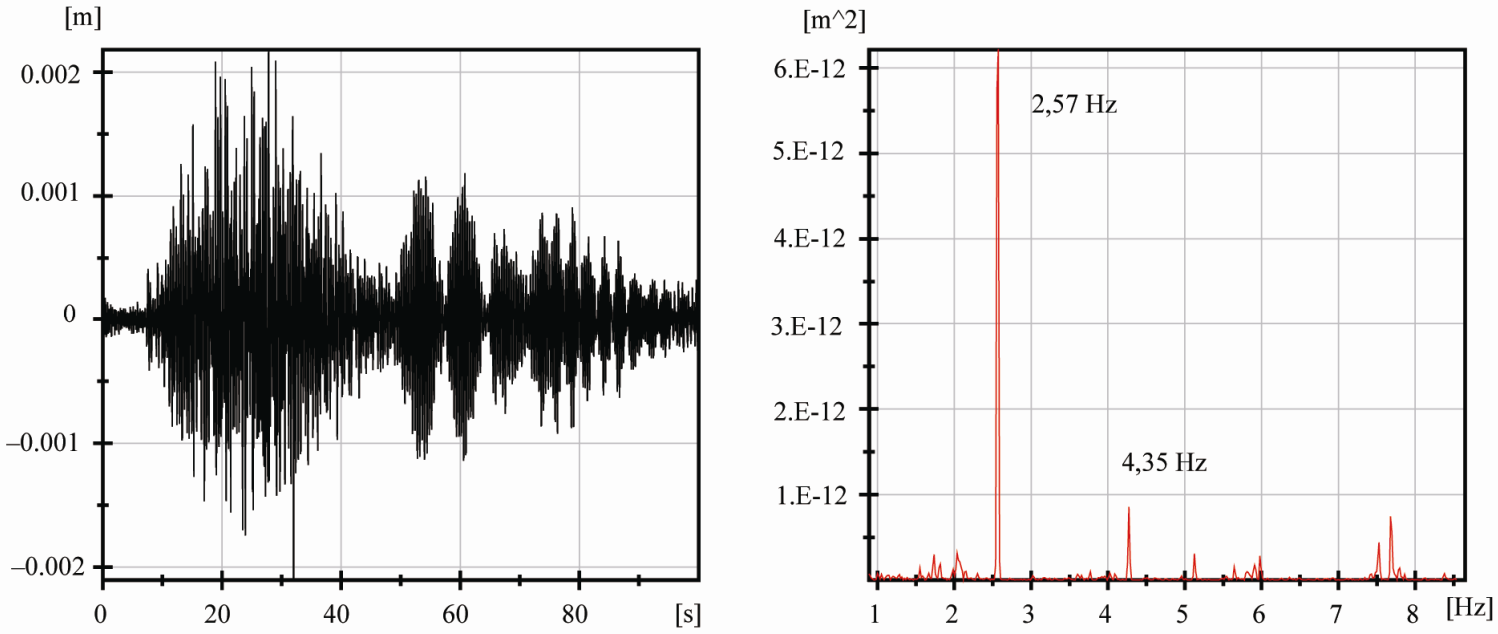

Figure 8. Time response of flexural deflection $w_{(t)}$ and power spectrum of response $\operatorname{SD}(f)$ in point BK1.
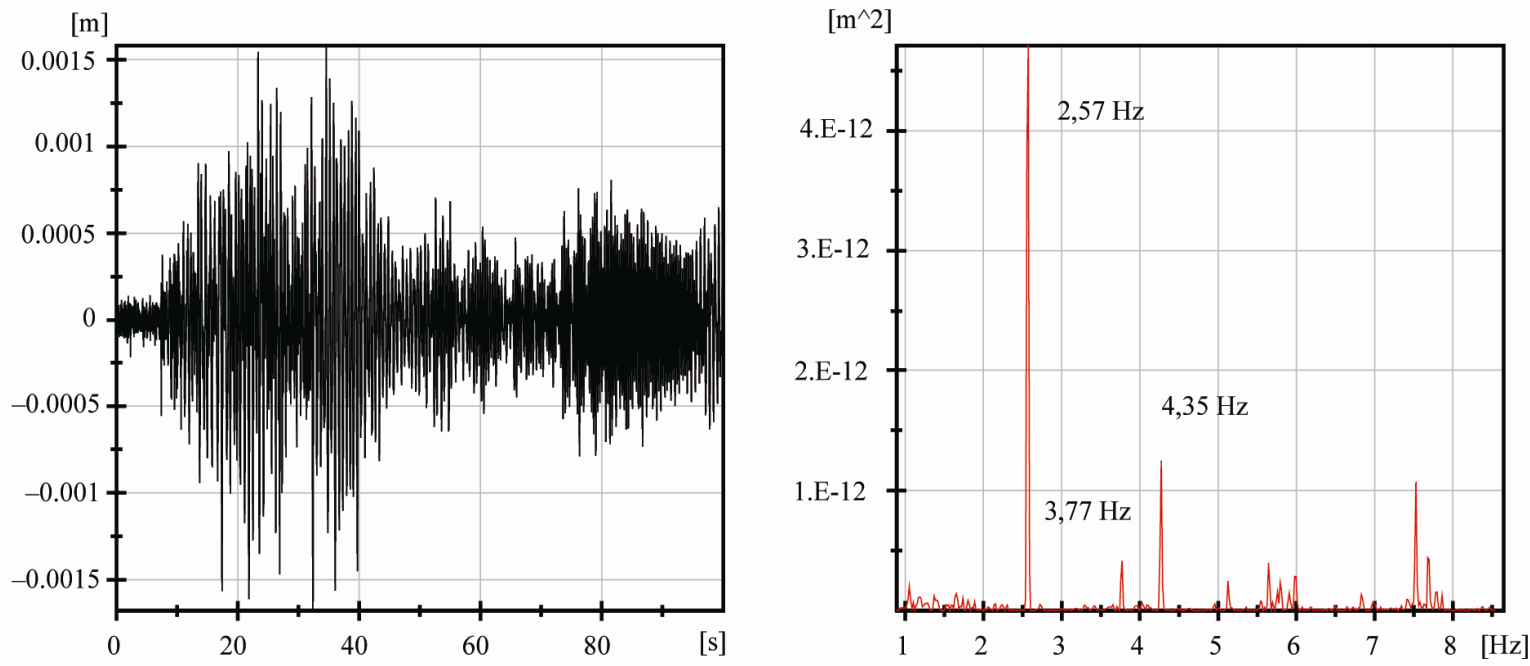

Figure 9. Time response of flexural deflection $w_{(t)}$ and power spectrum of response $S_{D}(f)$ in point BK2, smooth run Nr. 1, (BA $-\mathrm{PE}), 1 \times$ MAN, $v=8 \mathrm{~km} / \mathrm{h}, w_{\max }=0.001581 \mathrm{~m}$.
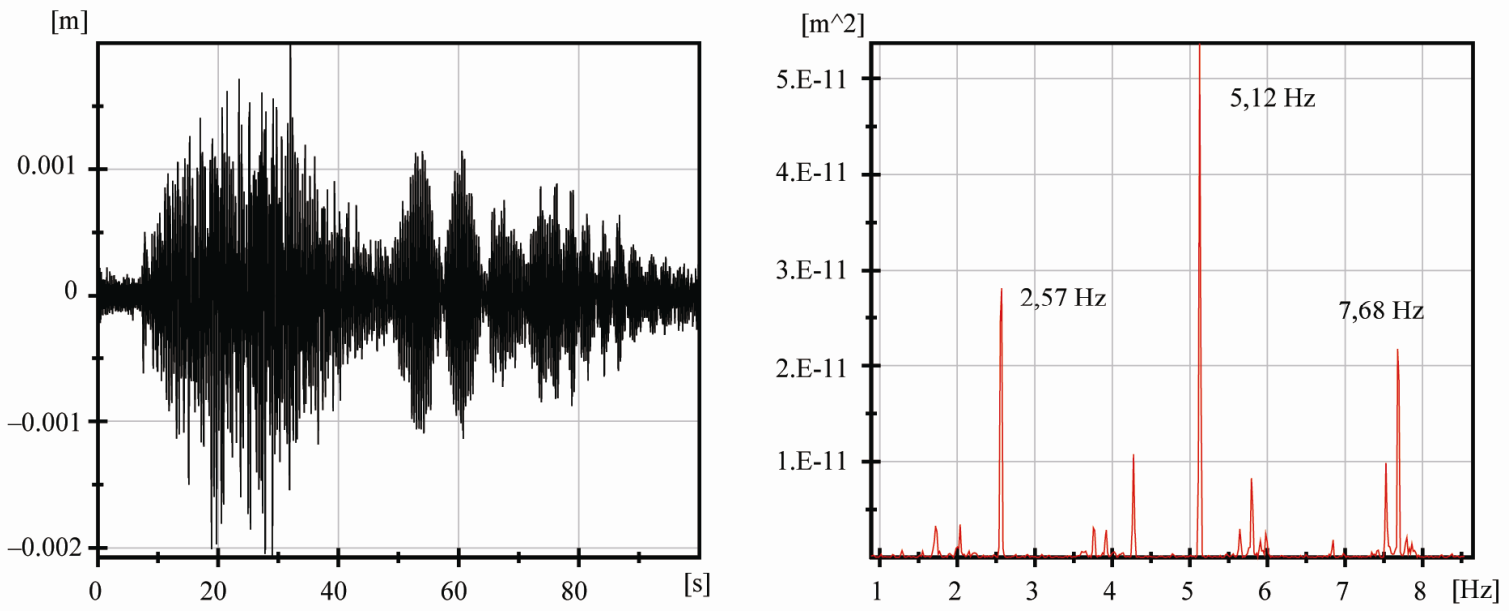

Figure 10. Time response of flexural deflection $w_{(t)}$ and power spectrum of response $S_{D}(f)$ in point BK3. 
Table 4. Dominant frequencies of the bridge.

\section{OLD BRIDGE CROSSING DANUBE IN BRATISLAVA}

\begin{tabular}{|c|c|c|c|c|c|}
\hline \multirow{2}{*}{$\begin{array}{l}\text { Run } \\
\text { Nr. }\end{array}$} & \multirow{2}{*}{ Direction } & \multirow{2}{*}{$\begin{array}{l}\text { Speed } \\
{[\mathrm{km} / \mathrm{h}]}\end{array}$} & \multicolumn{2}{|r|}{ Smooth runs } & direction \\
\hline & & & vertical (BK1) & $\begin{array}{l}\text { horizontal } \\
\text { (BK2) }\end{array}$ & $\begin{array}{l}\text { vertical } \\
\text { (BK3) }\end{array}$ \\
\hline 1 & $\mathrm{BA}-\mathrm{PE}$ & 8 & $\begin{array}{c}2.57 \\
(0.878 ; 4.35)\end{array}$ & $\begin{array}{c}2.57 \\
(3.77 ; 4.35)\end{array}$ & $\begin{array}{c}5.12(2.58 ; \\
4.35)\end{array}$ \\
\hline 2 & PE - BA & 9 & $\begin{array}{c}2.57 \\
(4.28,5.81)\end{array}$ & $\begin{array}{c}2.57 \\
(3.77 ; 4.35)\end{array}$ & $\begin{array}{c}2.57 \\
(2.06 ; 5.12)\end{array}$ \\
\hline 3 & $\mathrm{BA}$ - PE & 18 & $\begin{array}{c}2.57 \\
(1.33,5.81)\end{array}$ & $\begin{array}{c}2.57 \\
(3.77 ; 4.35)\end{array}$ & $\begin{array}{c}2.57 \\
(1.65 ; 5.51)\end{array}$ \\
\hline 4 & $\mathrm{PE}$ & 18 & $\begin{array}{c}1.17 \\
(1.29 ; 2.57)\end{array}$ & $\begin{array}{c}1.44 \\
(1.14 ; 2.57)\end{array}$ & $\begin{array}{c}5.13 \\
(2.58 ; 4.70)\end{array}$ \\
\hline 5 & BA & 27 & $\begin{array}{c}1.16 \\
(2.57 ; 1.76)\end{array}$ & $\begin{array}{c}1.16 \\
(2.57 ; 4.35)\end{array}$ & $\begin{array}{l}2.57(1.16 \\
4.27)\end{array}$ \\
\hline 6 & PE - BA & 27 & $\begin{array}{c}2.57 \\
(8.73 ; 5.12 ; 4.35)\end{array}$ & $\begin{array}{c}4.35 \\
(2.57 ; 35.12)\end{array}$ & $2.57(0.33)$ \\
\hline 7 & BA - PE & 34 & $\begin{array}{c}2.57 \\
(1.16 ; 1.58)\end{array}$ & $\begin{array}{c}1.34 \\
(2.57 ; 5.14)\end{array}$ & $\begin{array}{c}2.57 \\
(5.14 ; 4.35)\end{array}$ \\
\hline 8 & BA & 39 & $\begin{array}{c}2.57 \\
(3.02 ; 8.5 ; 5.12)\end{array}$ & $\begin{array}{c}2.57 \\
(1.15 ; 4.14)\end{array}$ & $\begin{array}{c}2.57 \\
(4.70 ; 5.12)\end{array}$ \\
\hline 9 & $\mathrm{~B}$ & 54 & $\begin{array}{c}1.99 \\
(1.15 ; 2.57)\end{array}$ & $\begin{array}{c}1.79 \\
(1.15 ; 2.57)\end{array}$ & $\begin{array}{c}2.57 \\
(1.15 ; 4.71)\end{array}$ \\
\hline 10 & PE - BA & 45 & 2.57 (4.14; 6.66) & $\begin{array}{c}2.57 \\
(4.14 ; 6.66)\end{array}$ & $\begin{array}{l}2.57 \\
(5.12)\end{array}$ \\
\hline 11 & BA - PE & 54 & $\begin{array}{c}2.57 \\
(1.99 ; 3.0)\end{array}$ & $\begin{array}{c}5.12 \\
(2.57 ;)\end{array}$ & $\begin{array}{c}2.57 \\
(6.69 ; 4.66)\end{array}$ \\
\hline 12 & $\mathrm{PE}$ & 54 & $\begin{array}{c}2.30 \\
(2.57 ; 3.03)\end{array}$ & $\begin{array}{c}2.57 \\
(1.83 ; 4.24)\end{array}$ & $\begin{array}{c}5.13 \\
(2.57 ; 6.50)\end{array}$ \\
\hline 13 & $\mathrm{BA}$ & 45 & $\begin{array}{c}2.57 \\
(1.83 ; 2.99)\end{array}$ & $\begin{array}{l}2.57(1.83 \\
4.24)\end{array}$ & $\begin{array}{c}0.867 \\
(2.57 ; 6.50)\end{array}$ \\
\hline 14 & PE - BA & 64 & $\begin{array}{c}1.39 \\
(1.57 ; 2.03)\end{array}$ & $\begin{array}{c}1.31 \text { (1.58; } \\
1.80)\end{array}$ & $\begin{array}{c}1.21 \\
(1.85 ; 2.57)\end{array}$ \\
\hline
\end{tabular}

spectra for specification of dominant natural frequencies for flexure in vertical and horizontal direction as well as in torsion. The comparison calculation vs measurement was made without and with consideration of corrosion damages.

In addition spectral analysis was made in order to obtain stochastic parameters for the assessment. The spectral analysis was used for specification of coherence functions of the bridge.

All above parameters were adopted in approach developed for dynamic testing and assessment of bridges.

\section{Acknowledgements}

Authors are indebted to the Slovak Scientific Agency VEGA for suggesting and supporting of above research in scope of scientific grants submitted.

\section{REFERENCES}

[1] L. Da Vinci, “Manuscript RL 19115v; K/P 114r,” Royal Library, Windsor Castle, Windsor, ca. 1500.

[2] P. Paultre, O. Challal and J. Proux, "Bridge Dynamics and Dynamic Amplification Factors - A Review of Ana- lytical and Experimental Findings," Canadian Journal of Civil Engineering, Vol. 19, No. 2, 1992, pp. 260-278. doi:10.1139/192-032

[3] J. Proulx, D. Hébert and P. Poultre, "Dynamic bridge testing in Quebec," Proceedings of International Conference Structural Dynamics-EURODYN'93, Trondheim, Vol. 2, 1993, pp. 943-950.

[4] J. Bencat, "Dynamic Testing of the Old Bridge Crossing Danube in Bratislava,” Technical Report Nr. 6-3-21/SvF/06, TU Zilina, 2008.

[5] A. Tesar, "Testing of the Old Bridge Crossing Danube in Bratislava,” Technical Report, 2001.

[6] A. Tesar, "Transfer Matrix Method,” Kluwer Academic Publishers, Dordrecht/Boston/London, 1988.

[7] R. Razani, "Behaviour of Fully Stressed Design of Structures and Its Relationship to Minimum-Weight Design," Journal Amer. Inst. Aeron. Astron., Vol. 3, 1965, pp. 2262-2268.

[8] R. L. Fox, "Optimization Methods for Engineering Design,” Addison-Wesley Publishing Group, Reading, 1971

[9] U. Kirsch, "Synthesis of Structural Geometry Using Approximate Concepts," Composite Structures, Vol. 15, 1982, pp. 103-111.

[10] L. A. Schmit and H. Miura, "A New Structural Analysis/Synthesis Capability-Access," American Institute of Aeronautics and Astronautics Journal, Vol. 14, No. 5, 1975, pp. 661-671.

[11] A. Tesar, "Synthesis in Optimisation of Shell Bridge Structures,” Building Research Journal, Vol. 35, 1985, pp. 812-824.

[12] J. Rechenberg, “Evolutionsstrategie,” Reihe Problemata 15, F. Frommann Verlag, Stuttgart, 1977.

[13] A. Tesar, "Load-Bearing Control of Slender Bridges," International Journal for Numerical Methods in Engineering, Vol. 62, No. 7, 2005, pp. 924-936. doi:10.1002/nme.1213

[14] A. Tesar, et al., "Ultimate Response of Bionics Shells," Structural Engineering and Mechanics, Vol. 14, No. 2, 2002, pp. 135-150.

[15] A. Tesar, "Bionics and Fractal Configurations in Structural Engineering,” International Journal for Numerical Methods in Engineering, Vol. 68, No. 7, 2006, pp. 790807. doi:10.1002/nme.1734

[16] S. C. Burgess, "Multi-Functioning and Multi-Optimization in Feathers," International Journal of Design \& Nature, Vol. 1, No. 1, 2007, pp. 1-10.

[17] J. Bencat, "Dynamic Testing of the Old Bridge Crossing Danube in Bratislava," Technical Report Nr. 6-3/SvF/06, TU Zilina, 2006.

[18] J. Bendat and A. Piersol, "Engineering Applications of Correlation and Spectral Analysis," John Wiley and Sons, Inc., New York, 1980.

[19] J. Melcer, "Dynamic Testing of the Old Bridge Crossing Danube in Bratislava," Report Nr.6/34/SvF/05, TU Zilina, 2005. 